\title{
Multi-modal Network Representation Learning
}

\author{
Chuxu Zhang ${ }^{1}$, Meng Jiang ${ }^{2}$, Xiangliang Zhang ${ }^{3}$, Yanfang $\mathrm{Ye}^{4}$, Nitesh V. Chawla ${ }^{2}$ \\ ${ }^{1}$ Brandeis University, ${ }^{2}$ University of Notre Dame \\ ${ }^{3}$ King Abdullah University of Science and Technology, ${ }^{4}$ Case Western Reserve University \\ chuxuzhang@brandeis.edu,\{mjiang2,nchawla\}@nd.edu,xiangliang.zhang@kaust.edu.sa,yanfang.ye@case.edu
}

\begin{abstract}
In today's information and computational society, complex systems are often modeled as multi-modal networks associated with heterogeneous structural relation, unstructured attribute/content, temporal context, or their combinations. The abundant information in multi-modal network requires both a domain understanding and large exploratory search space when doing feature engineering for building customized intelligent solutions in response to different purposes. Therefore, automating the feature discovery through representation learning in multi-modal networks has become essential for many applications. In this tutorial, we systematically review the area of multi-modal network representation learning, including a series of recent methods and applications. These methods will be categorized and introduced in the perspectives of unsupervised, semi-supervised and supervised learning, with corresponding real applications respectively. In the end, we conclude the tutorial and raise open discussions. The authors of this tutorial are active and productive researchers in this area.
\end{abstract}

\section{KEYWORDS}

Multi-modal networks, Network representation learning, Deep learning

\section{ACM Reference Format:}

Chuxu Zhang ${ }^{1}$, Meng Jiang ${ }^{2}$, Xiangliang Zhang ${ }^{3}$, Yanfang Ye ${ }^{4}$, Nitesh V. Chawla $^{2}$. 2020. Multi-modal Network Representation Learning. In Proceedings of the 26th ACM SIGKDD Conference on Knowledge Discovery and Data Mining (KDD '20), August 23-27, 2020, Virtual Event, CA, USA. ACM, New York, NY, USA, 2 pages. https://doi.org/10.1145/3394486.3406475

\section{INTRODUCTION}

Complex systems such as social media, cybersecurity system, or chemical synthesis, are often modeled as multi-modal networks. Different from the general homogeneous networks (e.g., social network), they are associated with complexity in heterogeneous structural relation (e.g., heterogeneous network with multi-typed nodes and relations), unstructured attribute/content (e.g., attributed network with node/edge attributes), temporal context (e.g., dynamic network), or their combination (e.g., attributed heterogeneous network). Automating the feature discovery through representation learning can largely reduce labor-consuming feature engineering

Permission to make digital or hard copies of part or all of this work for personal or classroom use is granted without fee provided that copies are not made or distributed for profit or commercial advantage and that copies bear this notice and the full citation on the first page. Copyrights for third-party components of this work must be honored. For all other uses, contact the owner/author(s).

KDD '20, August 23-27, 2020, Virtual Event, CA, USA

(c) 2020 Copyright held by the owner/author(s).

ACM ISBN 978-1-4503-7998-4/20/08.

https://doi.org/10.1145/3394486.3406475 activity which requires both domain understanding and large exploratory search when addressing various problems in those complex data. In recent years, representation learning in multi-modal networks has been a popular research topic with practical significance in many applications such as recommendation/search, behavior prediction, anomaly detection, etc. Therefore, we are motivated to organize this tutorial to review the state-of-the-art work for multi-modal network representation learning and illustrate how they can tackle real-world problems. Aligned with machine learning strategies, representation learning for multi-modal networks can be categorized as supervised, semi-supervised, and unsupervised learning methods, according to the usage of available supervisory information. In this tutorial, we systematically review recent studies in these directions and introduce corresponding applications.

- Supervised methods and applications. Supervisory information (e.g., relation pairs, node class labels) is extremely helpful on guiding the representation learning in multi-modal networks. The supervised approaches use ground-truth labeled information to develop task guided models and learn customized node representations for specific tasks in multi-modal networks. The related work brings impact to various applications including relevance search [21, 25], recommender systems [2, 4, 19, 20, 24], user profiling [12], event detection [8], behavior prediction [11, 15, 16], and malware and anomaly detection $[6,7,9,18]$.

- Semi-supervised methods and applications. Supervising information is often expensive to collect. Therefore, semi-supervised representation learning for multi-model networks becomes a highly demanding, while barely explored technique. It is expected that the model is trained with a small portion of labeled nodes and plenty of unlabeled nodes. The learned node representation naturally serves well for node classification [1], multi-label classification [1], graph alignment [13, 14], and so on.

- Unsupervised methods and applications. Without any supervised information, the unsupervised models aim to learn task independent node representations (without ground-truth label) for different tasks (e.g., node classification) in multi-modal networks. The related work includes heterogeneous network embedding [3, 5, 10, 23], heterogeneous graph neural network [22], temporal/dynamic/evolutionary graph neural network [17], and graph neural networks for unsupervised anomaly detection [26].

The intended audiences are students, researchers, and practitioners who are new to this topic or who already have some experience in data mining, machine learning, and network science.

\section{SIMILAR EVENTS AND SOCIETAL IMPACTS}

Several similar tutorials have been given at related conferences: (i) Peng Cui et al., Modeling Data With Networks + Network Embedding: Problems, Methodologies and Frontiers, at KDD 2018; (ii) Jian Tang and William L. Hamilton, Graph Representation Learning, at 
AAAI 2019; (iii) Jie Tang and Yuxiao Dong, Representation Learning on Networks, at WWW 2019. This tutorial is different from them in two main aspects: (1) it focuses on the study for representation learning in multi-modal networks with complexity in structure, content, dynamics, or their combinations, while the previous tutorials review work for general networks; (2) it covers the most recently techniques, many of which are not included in previous tutorials. This tutorial will provide a good resource and inspirations for researchers and practitioners.

\section{OUTLINE}

The outline and schedule of the 3-hour tutorial is as follows.

\begin{tabular}{l}
\hline 1. Introduction and Overview (20 mins) \\
1.1 Multi-modal network representation learning \\
1.2 Tutorial organization \\
\hline 2. Supervised Methods and Applications (60 mins) \\
2.1 Task-guided relation learning \\
2.2 User and behavior context learning \\
2.3 Malware detection \\
2.4 Abnormal user identification \\
\hline 3. Semi-supervised Methods and Applications (30 mins) \\
3.1 Semi-supervised attributed network embedding \\
3.2 Semi-supervised graph alignment \\
\hline 4. Unsupervised Methods and Applications (50 mins) \\
4.1 Heterogeneous network embedding \\
4.2 Heterogeneous graph neural network \\
4.3 Temporal/dynamic/evolutionary graph neural network \\
4.4 Graph neural network for unsupervised anomaly detection \\
\hline 5. Conclusions and Discussions (20 mins) \\
\hline
\end{tabular}

\section{TUTORS' BIOGRAPHY}

Chuxu Zhang is an Assistant Professor in the Department of Computer Science at the Brandeis University. His research interests are data mining, machine learning, and their applications in graph/network mining, recommendation/user modeling, natural language processing and science domains (e.g., chemistry). His work have appeared in KDD, WWW, AAAI, IJCAI, and so on.

Meng Jiang is an Assistant Professor in the Department of Computer Science and Engineering at the University of Notre Dame. His research interests include data mining, machine learning, and information extraction. His research work focuses on computational behavior modeling. He has published over 50 conference and journal papers of the topics. His work was recognized as ACM SIGKDD 2014 Best Paper Finalist. He has delivered six tutorials in conferences such as KDD, SIGMOD, WWW, CIKM, and ICDM. He is the recipient of Notre Dame Global Gateway Faculty Award.

Xiangliang Zhang is an Associate Professor of Computer Science and directs the Machine Intelligence and Knowledge Engineering (http://mine.kaust.edu.sa) group at KAUST, Saudi Arabia. Her research mainly focuses on learning from complex and large-scale streaming and graph data. She has published over 130 research papers in referred international journals and conference proceedings, including TKDE, SIGKDD, AAAI, IJCAI, ICDM, VLDB J, ICDE etc. She is selected and invited to deliver an Early Career Spotlight talk at IJCAI-ECAI 2018.
Yanfang (Fanny) Ye is the Theodore L. and Dana J. Schroeder Associate Professor in the Department of Computer and Data Sciences (CDS) at Case Western Reserve University (CWRU). Her research mainly focuses on data mining, machine learning, cybersecurity and health intelligence. She recently received the prestigious NSF Career Award (2019) and IJCAI Early Career Spotlight (2019), the AICS 2019 Challenge Problem Winner, the ACM SIGKDD 2017 Best Paper Award and ACM SIGKDD 2017 Best Student Paper Award (Applied Data Science Track), and so on.

Nitesh V. Chawla is the Frank M. Freimann Professor in the Department of Computer Science and Engineering at the University of Notre Dame and the Director of the Center for Network and Data Science (CNDS) at Notre Dame. His research focuses on machine learning, AI and network science fundamentals and interdisciplinary applications. His papers have received several best paper nominations and awards. He is also the recipient of several awards and honors including IEEE CIS Outstanding Early Career Award, the IBM Watson Faculty Award, and so on.

\section{REFERENCES}

[1] Uchenna Akujuobi, Yufei Han, Qiannan Zhang, and Xiangliang Zhang. 2019. Collaborative Graph Walk for Semi-supervised Multi-Label Node Classification. In ICDM.

[2] Basmah Altaf, Uchenna Akujuobi, Lu Yu, and Xiangliang Zhang. 2019. Dataset Recommendation via Variational Graph Autoencoder. In ICDM.

[3] Xia Chen, Guoxian Yu, Jun Wang, Carlotta Domeniconi, Zhao Li, and Xiangliang Zhang. 2019. ActiveHNE: Active Heterogeneous Network Embedding. In IfCAI.

[4] Yujun Chen, Yuanhong Wang, Yutao Zhang, Juhua Pu, and Xiangliang Zhang. 2019. AMENDER: an Attentive and Aggregate Multi-layered Network for Dataset Recommendation. In $I C D M$.

[5] Yuxiao Dong, Nitesh V Chawla, and Ananthram Swami. 2017. metapath2vec: Scalable Representation Learning for Heterogeneous Networks. In KDD.

[6] Yujie Fan, Shifu Hou, Yiming Zhang, Yanfang Ye, and Melih Abdulhayoglu. 2018. Gotcha-Sly Malware! Scorpion A Metagraph2vec Based Malware Detection System. In KDD.

[7] Yujie Fan, Yiming Zhang, Yanfang Ye, and Xin Li. 2018. Automatic Opioid User Detection from Twitter: Transductive Ensemble Built on Different Meta-graph Based Similarities over from Twitter: Transductive Ensemble Built on

[8] Huan Gui, Jialu Liu, Fangbo Tao, Meng Jiang, Brandon Norick, Lance Kaplan, and Jiawei Han. 2017. Embedding learning with events in heterogeneous information networks. TKDE (2017).

[9] Shifu Hou, Yanfang Ye, Yangqiu Song, and Melih Abdulhayoglu. 2017. Hindroid: An intelligent android malware detection system based on structured heterogeneous information network. In $K D D$.

[10] Meng Jiang, Peng Cui, Fei Wang, Xinran Xu, Wenwu Zhu, and Shiqiang Yang. 2014. Fema: flexible evolutionary multi-faceted analysis for dynamic behavioral pattern discovery. In KDD.

[11] Meng Jiang, Peng Cui, Nicholas Jing Yuan, Xing Xie, and Shiqiang Yang. 2016. Little is much: Bridging cross-platform behaviors through overlapped crowds. In AAAI.

[12] Zaiqiao Meng, Shangsong Liang, Hongyan Bao, and Xiangliang Zhang. 2019. Co-Embedding Attributed Networks. In WSDM.

[13] Shichao Pei, Lu Yu, Robert Hoehndorf, and Xiangliang Zhang. 2019. Semi-Supervised Entity Alignment via Knowledge Graph Embedding with Awareness of Degree Difference. In WWW.

[14] Shichao Pei, Lu Yu, and Xiangliang Zhang. 2019. Improving Cross-lingual Entity Alignment via Optimal Transport. In IFCAI.

[15] Daheng Wang, Meng Jiang, Qingkai Zeng, Zachary Eberhart, and Nitesh V Chawla. 2018. Multi-type itemset embedding for learning behavior success. In KDD

[16] Daheng Wang, Tianwen Jiang, Nitesh V Chawla, and Meng Jiang. 2019. TUBE: Embedding Behavior Outcomes for Predicting Success. In $K D D$.

[17] Daheng Wang, Tong Zhao, Tianwen Jiang, Nitesh V Chawla, and Meng Jiang. 2020. Evolutionary Graph Neural Networks. In Under Review.

[18] Yanfang Ye, Shifu Hou, Lingwei Chen, Jingwei Lei, Wenqiang Wan, Jiabin Wang, Qi Xiong, and Fudong Shao. 2019. Out-of-sample node representation learning for heterogeneous graph in real-time android malware detection. In $I F C A I$.

[19] Lu Yu, Chuxu Zhang, Shangsong Liang, and Xiangliang Zhang. 2019. Multi-Order Attentive Ranking Model for Sequential Recommendation. In AAAI.

[20] Lu Yu, Chuxu Zhang, Shichao Pei, Guolei Sun, and Xiangliang Zhang. 2018. Walkranker: A unified pairwise ranking model with multiple relations for item recommendation. In AAAI.

[21] Chuxu Zhang, Chao Huang, Lu Yu, Xiangliang Zhang, and Nitesh V Chawla. 2018. Camel: Content-Aware and Meta-path Augmented Metric Learning for Author Identification.. In $W W W$.

[22] Chuxu Zhang, Dongjin Song, Chao Huang, Ananthram Swami, and Nitesh V. Chawla. 2019. Heterogeneous Graph Neural Network. In $K D D$.

[23] Chuxu Zhang, Ananthram Swami, and Nitesh V Chawla. 2019. SHNE: Representation Learning for Semantic-Associated Heterogeneous Networks. In WSDM.

[24] Chuxu Zhang, Lu Yu, Yan Wang, Yan Wang, and Xiangliang Zhang. 2017. Collaborative User Network Embedding for Social Recommender Systems. In SDM.

[25] Chuxu Zhang, Lu Yu, Xiangliang Zhang, and Nitesh V Chawla. 2018. Task-Guided and Semantic-Aware Ranking for Academic Author-Paper Correlation Inference.. In IFCAI.

[26] Tong Zhao, Chuchen Deng, Kaifeng Yu, Tianwen Jiang, Daheng Wang, and Meng Jiang. 2020. Learning with Bipartite Graph Anomaly Losses. In Under Review. 